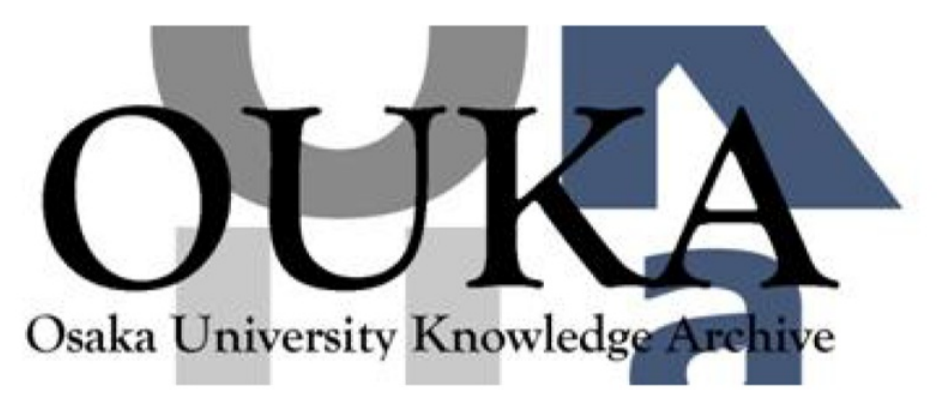

\begin{tabular}{|c|l|}
\hline Title & $\begin{array}{l}\text { Modulated photoconductivity study of electron } \\
\text { drift mobility in amorphous silicon }\end{array}$ \\
\hline Author(s) & Hattori, K.; Iida, M.; Hirao, T. et al. \\
\hline Citation & Journal of Applied Physics. $87(6)$ p. 2901-p. 2909 \\
\hline Issue Date & $2000-03-15$ \\
\hline oaire:version & VoR \\
\hline URL & https://hdl. handle. net/11094/2925 \\
\hline rights & \\
\hline Note & \\
\hline
\end{tabular}

Osaka University Knowledge Archive : OUKA

https://ir. Library. osaka-u. ac. jp/

0saka University 


\title{
Modulated photoconductivity study of electron drift mobility in amorphous silicon
}

\author{
K. Hattori, ${ }^{a)}$ M. lida, T. Hirao, and H. Okamoto \\ Department of Physical Science, Graduate School of Engineering Science, Osaka University, Toyonaka, \\ Osaka 560-8531, Japan
}

(Received 2 August 1999; accepted for publication 14 December 1999)

\begin{abstract}
The modulated photoconductivity technique, a convenient means of measuring the drift mobility of photocarriers, has been applied to investigate carrier transport in hydrogenated amorphous silicon. The frequency resolved spectra of drift mobility that can be obtained from the measurements were analyzed in accordance with a generalized transport model that included possible carrier interactions between localized states through tunneling transitions. Theory suggests that a tunneling-assisted thermalization of nonequilibrium carriers appreciably affects the transport process. The experimental results are reasonably accounted for by the introduced model, leading to quantitative assessments for transport mechanisms. (C) 2000 American Institute of Physics.
\end{abstract}

[S0021-8979(00)08006-3]

\section{INTRODUCTION}

A fundamental property of amorphous semiconductors is the presence of localized states. The interactions of excess carriers with localized states have a significant influence on the transport process as clearly manifested in transient photocurrent measurements with pulsed excitation, such as a time-of-flight (TOF) measurement in a sandwich configuration. ${ }^{1}$ The investigation of photocarrier transport has greatly contributed to the understanding of these materials. In the TOF experiment, a sheet of carriers is created near one surface by a brief flash of strongly absorbed light. A voltage applied across the sample causes the carriers to drift to the opposite surface. The propagation of carriers is monitored by the current induced in an external circuit. The TOF transport in amorphous semiconductors is frequently described by introducing the concept of drift mobility that is defined as an effective mobility averaged over all the carriers involved in transport. The drift mobility of experimental concern is that of carriers in a highly nonequilibrium distribution that relaxes as the carriers traverse the sample, and is in essence time dependent. The present prevailing theory for the transient drift mobility phenomena is the multiple trapping model, ${ }^{2}$ which considers that carriers move by repeatedly transitioning between extended and localized states, becoming trapped in localized states then releasing from localized states to extended states.

In this study, a frequency resolved technique based on modulated photoconductivity (MPC) in a coplanar geometry has been applied to investigate the drift mobility of photocarriers and their interactions with localized states. A detailed discussion is given for interpreting the frequency domain measurements of drift mobility in accord with a generalized transport model. The model takes into account possible carrier interactions between localized states through tunneling transitions in the context of a mean field approximation. The theoretical consideration suggests that a

${ }^{a)}$ Electronic mail: hattori@ee.es.osaka-u.ac.jp tunneling-assisted thermalization of nonequilibrium carriers takes part in the transport process to an appreciable degree. The developed model accounts for the experimental results obtained for hydrogenated amorphous silicon $(a-\mathrm{Si}: \mathrm{H})$, yielding quantitative assessments of the transport properties of this material.

\section{THEORY AND ANALYSIS}

\section{A. Phenomenological formulation}

We here consider an amorphous semiconductor layer in a coplanar configuration uniformly illuminated by a weakly absorbed band-gap light. The extended band states and the localized gap states are both populated with photogenerated excess carriers. The charge neutrality requirement imposes

$$
N=n+\sum_{i} n_{i}=p+\sum_{k} p_{k},
$$

where $N$ is the total concentration of charge carriers, $n$ and $p$ refer to the concentrations of electrons in the conduction band states and holes in the valence band states, respectively, $n_{i}$ the concentration of electrons trapped at the localized states of energy $\epsilon_{i}$, and $p_{k}$ the concentration of holes trapped at the localized states of energy $\epsilon_{k}$. With electron and hole currents being spatially uniform, the continuity equation is simply expressed by

$$
\frac{d}{d t} N=G-R,
$$

where $G$ denotes the generation rate and $R$ the recombination rate. For an excitation light sinusoidally varying in time such that

$$
G=G_{0}+\Delta G \cos (\omega t),
$$

a first-order solution for carrier concentration is given by

$$
N=N_{0}+\operatorname{Re}[\Delta N \exp (i \omega t)],
$$

where $i^{2}=-1, N_{0}=n_{0}+\sum_{i} n_{i 0}=p_{0}+\sum_{k} p_{k 0}$ is the steadystate concentration generated by $G_{0}$, and $\Delta N=\Delta n+\sum_{i} \Delta n_{i}$ 
$=\Delta p+\sum_{k} \Delta p_{k}$ is the complex number that gives amplitude and phase of the sinusoidal part of concentration. The expression assumes a linear response to the modulated illumination, which can be generally validated for a small perturbation $\Delta G \ll G_{0}$. In such a linear response regime, the recombination rate is represented in a phenomenological manner by

$$
R=R_{0}+\operatorname{Re}\left[\frac{\Delta N}{\bar{\tau}} \exp (i \omega t)\right] .
$$

The average lifetime for all the charge carriers $\bar{\tau}$ defined above permits formulating the modulated component of carrier concentration as

$$
\Delta N=\frac{\Delta G \bar{\tau}}{1+i \omega \bar{\tau}}
$$

The transport of carriers may occur via the extended states as well as the localized states. A comprehensive representation for MPC, taking into account all the possible channels, is given by

$$
\Delta \sigma=q\left[\mu_{c} \Delta n+\mu_{\nu} \Delta p+\sum_{i} \mu_{c i} \Delta n_{i}+\sum_{k} \mu_{\nu k} \Delta p_{k}\right],
$$

where $q$ denotes the unit electronic charge, $\mu_{c, \nu}$ the mobilities of electrons and holes in the extended states, respectively, and $\mu_{c i, v k}$ the mobilities of electrons and holes in the localized states, respectively. It may be convenient here to introduce the drift mobility defined as

$$
\bar{\mu}=\frac{\mu_{c} \Delta n+\mu_{\nu} \Delta p+\sum_{i} \mu_{c i} \Delta n_{i}+\sum_{k} \mu_{\nu k} \Delta p_{k}}{\Delta N} .
$$

Combining Eqs. (6)-(8), we obtain a concise expression

$$
\Delta \sigma=\frac{q \Delta G \bar{\mu} \bar{\tau}}{1+i \omega \bar{\tau}}
$$

The MPC parameters $\bar{\mu}$ and $\bar{\tau}$ introduced above, being functions of frequency, generalize the small-signal parameters employed for formulation of the steady-state photocarrier grating transport. ${ }^{3,4}$ Our MPC theory is quite general at this stage in the sense that it does not depend on the details of the transport and recombination mechanisms.

The following part of discussion relies on a regional approximation with respect to frequency. In the high frequency regime where the condition $\omega|\bar{\tau}| \gg 1$ is met, one obtains

$$
\Delta \sigma=\frac{q \Delta G \bar{\mu}}{i \omega},
$$

indicating that the MPC behavior is free from the effect of the recombination described by $\bar{\tau}$ and controlled only by the drift mobility $\bar{\mu}$. The drift mobility can be therefore determined from the measurement of the high frequency MPC in terms of the relationship

$$
\bar{\mu}=\frac{i \omega \Delta \sigma}{q \Delta G} .
$$

An equivalent formula has been deduced in previous MPC studies using a photomixing technique. ${ }^{5}$ On the other hand, Eq. (9) is reduced to

$$
\Delta \sigma=q \Delta G \bar{\mu} \bar{\tau}
$$

in the low frequency regime where $\omega|\bar{\tau}| \ll 1$. Expected in this extreme limit is that the carrier system stays in quasithermal equilibrium during modulation and thereby the transport parameters $\bar{\mu}$ and $\bar{\tau}$ become frequency independent. In Appendix A, the plausibility of MPC behavior suggested above is demonstrated by model calculations.

\section{B. Rate equations}

In this section, we will deal with drift mobility for the case in which electrons are the predominant type of carriers in photoconduction, so as to accommodate photocarrier transport in $a$ - $\mathrm{Si}: \mathrm{H}$. This considerably reduces complications in the theoretical analysis and thereby makes the discussion more straightforward. It is also assumed that contributions due to hopping transport in the general formula (8) are minor. A justification of this neglect is given by means of numerical calculation in Appendix B. The drift mobility is then simply rewritten as

$$
\bar{\mu}=\mu_{c} \frac{\Delta n}{\Delta N} .
$$

Even in such a simple case, contributions by tunneling processes cannot be completely excluded from consideration. There is a possibility that tunneling interactions between localized states may affect nonequilibrium occupation, on which the population ratio $\Delta n / \Delta N$ and the drift mobility $\bar{\mu}$ depend. We will address this point in the following.

The starting point for the analysis is the set of rate equations for a distribution of localized states with densities $N_{i}$ and energies $\epsilon_{i}$,

$$
\begin{aligned}
\frac{d n}{d t}= & G-\sum_{i}\left[n C\left(N_{i}-n_{i}\right)-e_{i} n_{i}\right]-\frac{n-n^{e q}}{\tau}, \\
\frac{d n_{i}}{d t}= & n C\left(N_{i}-n_{i}\right)-e_{i} n_{i}-n_{i} \sum_{j \neq i} w_{i j}+\sum_{j \neq i} n_{j} w_{j i} \\
& -\frac{n_{i}-n_{i}^{e q}}{\tau_{i}} .
\end{aligned}
$$

All the localized states considered here have an identical efficiency of capturing a band electron, which is described by the coefficient $C$. The thermal emission of electrons from the localized states of energy $\epsilon_{i}$ to the conduction band states above $\epsilon_{c}$ occurs at the rate $e_{i}$ given by

$$
e_{i}=N_{c} C \exp \left[-\frac{\epsilon_{c}-\epsilon_{i}}{k T}\right],
$$

where $k T$ is the thermal energy and $N_{c}$ is the effective density of conduction band states. Equations (14) and (15) are written with monomolecular recombination terms containing lifetime parameters $\tau$ and $\tau_{i}$. They constitute the rate of charge recombination represented by

$$
R=\frac{n-n^{e q}}{\tau}+\sum_{i} \frac{n_{i}-n_{i}^{e q}}{\tau_{i}},
$$


where the superscript $e q$ refers to the value in thermal equilibrium. Considering a particular mechanism for recombination is not essential to the analysis of high frequency MPC or the drift mobility determined from it, but is necessitated for quantitative arguments on the low-frequency MPC and the steady-state photoconductivity. The tunneling rate of an electron from the state $i$ to the state $j$, separated by a distance $r$ $=\left|\mathbf{r}_{j}-\mathbf{r}_{i}\right|$ and an energy $\epsilon_{i j}=\epsilon_{j}-\epsilon_{i}$, is expressed by ${ }^{6}$

$$
w_{i j}=\nu \exp \left[-\frac{2 r}{\alpha}-\frac{\theta\left(\epsilon_{i j}\right) \epsilon_{i j}}{k T}\right] \text {, }
$$

where $\nu$ is the frequency prefactor, $\alpha$ the localization radius, and $\theta(\epsilon)$ the unit step function. It is easily confirmed that Eqs. (14) and (15) are consistent with the continuity Eq. (2).

In order to make the rate equations given above tractable, we here use the average tunneling rate assessed in the framework of the mean field model that assumes each state is surrounded by the same average environment. ${ }^{7}$ The tunneling transitions preferentially take place between the nearest available neighbors. The distribution function of nearest neighbor separations $r$ from a particular occupied state is given by

$$
P_{j}(r)=4 \pi r^{2}\left(N_{j}-n_{j}\right) \exp \left[-\frac{4 \pi r^{3}\left(N_{j}-n_{j}\right)}{3}\right],
$$

for a randomly distributed collection of unoccupied states with density $N_{j}-n_{j}$. The configuration average is then

$$
\left\langle w_{i j}\right\rangle=\int w_{i j}(r) P_{j}(r) d r
$$

An essential requirement for localization is that the localization radius $\alpha$ is less than the mean distance $\left(3 / 4 \pi N_{j}\right)^{1 / 3}$ between the states, leading to the approximation

$$
\left\langle w_{i j}\right\rangle=C_{i j}\left(N_{j}-n_{j}\right),
$$

with

$$
C_{i j}=\nu \pi \alpha^{3} \exp \left[-\frac{\theta\left(\epsilon_{i j}\right) \epsilon_{i j}}{k T}\right] .
$$

In the mean field approach, Eq. (15) is consequently rewritten by

$$
\begin{aligned}
\frac{d n_{i}}{d t}= & n C\left(N_{i}-n_{i}\right)-e_{i} n_{i}-n_{i} \sum_{j \neq i} C_{i j}\left(N_{j}-n_{j}\right) \\
& +\left(N_{i}-n_{i}\right) \sum_{j \neq i} n_{j} C_{j i}-\frac{n_{i}-n_{i}^{e q}}{\tau_{i}} .
\end{aligned}
$$

A strong localization limit where $\alpha=0$ and equivalently $C_{i j}$ $=0$ deals with the conventional multiple trapping model that ignores any carrier interactions between localized states. It might be worthwhile to mention that the average tunneling rate given above meets the detailed balance requirement in thermal equilibrium such that

$$
f_{i}^{e q} C_{i j}\left(1-f_{j}^{e q}\right)=f_{j}^{e q} C_{j i}\left(1-f_{i}^{e q}\right),
$$

where $f_{i}^{e q}=\left\{1+\exp \left[\left(\epsilon_{i}-\epsilon_{F}\right) / k T\right]\right\}^{-1}$ is the Fermi-Dirac distribution with Fermi level $\epsilon_{F}$.
A linearization of Eqs. (14) and (23) for the modulated illumination Eq. (3) is easily achieved by collecting the first order terms in $\Delta n, \Delta n_{i}$, and $\Delta G$ and replacing the derivative by $i \omega$. The steady-state solutions $n_{0}$ and $n_{i 0}$ to be contained in the linearized equations should be considered carefully. Neglecting the recombination term $\left(n_{i 0}-n_{i}^{e q}\right) / \tau_{i}$, the steady-state occupancy $f_{i 0}=n_{i 0} / N_{i}$ is given by

$$
f_{i 0}=\frac{n_{0} C}{n_{0} C+e_{i}} .
$$

The occupation function $f_{i 0}$ is equivalent to the distribution $f_{i}^{e q}$ with the Fermi level $\epsilon_{F}$ replaced by the quasi-Fermi level $\epsilon_{c}-k T \ln \left(N_{c} / n_{0}\right)$. The following equality:

$$
f_{i 0} C_{i j}\left(1-f_{j 0}\right)=f_{j 0} C_{j i}\left(1-f_{i 0}\right) \text {, }
$$

then stands for tunneling transitions. This ensures that every tunneling term involved in the steady-state equation is canceled out and does not appear in the expression for $f_{i 0}$. For localized states lying in the vicinity of the band edge, like the conduction band tail in $a$-Si:H, we may expect that $n_{0} C$ $\ll e_{i}$ and thereby $f_{i 0} \ll 1$ under a moderate excitation. It should be noted that the assumption of the empty states is valid even if the recombination events described by $\tau_{i}$, which tend to reduce $f_{i 0}$, are taken into consideration. On the basis of these arguments, we finally reach the equations that represent the high frequency MPC

$$
\begin{aligned}
& \Delta n\left(i \omega+\sum_{i} C N_{i}\right)-\sum_{i} \Delta n_{i} e_{i}=\Delta G \\
& \Delta n C N_{i}-\Delta n_{i}\left(i \omega+e_{i}+\sum_{j \neq i} C_{i j} N_{j}\right)+\sum_{j \neq i} \Delta n_{j} C_{j i} N_{i}=0 .
\end{aligned}
$$

The solutions for Eqs. (27) and (28), as well as the drift mobility determined from them, will be investigated in a later section.

\section{Model calculation}

We here introduce the quantities defined as

$$
\begin{aligned}
& \hat{N}_{i}=\frac{N_{i}}{N_{c}}, \\
& \Delta \hat{f}_{i}=\frac{N_{c} \Delta n_{i}}{N_{i} \Delta n} .
\end{aligned}
$$

The normalized occupancy $\Delta \hat{f}_{i}$ is the ratio of occupancies $\Delta n_{i} / N_{i}$ and $\Delta n / N_{c}$ in the framework of the present rate equation analysis, where the distribution of extended states is equivalent to a single effective level with density $N_{c}$ and energy $\epsilon_{c}$. The drift mobility is formulated with these definitions as

$$
\frac{\bar{\mu}}{\mu_{c}}=\frac{1}{1+\sum_{i} \hat{N}_{i} \Delta \hat{f}_{i}} .
$$

In this expression, a direct connection between the drift mobility and the normalized occupancy is evident.

It is instructive to outline the analytic properties of the multiple trapping model assuming $\alpha=0$ before proceeding to 
more general cases. A simple analytical solution for the normalized occupancy is easily achieved in the strong localization limit to be

$$
\Delta \hat{f}_{i}=\frac{N_{c} C}{i \omega+e_{i}} .
$$

Readily understood is that a specific energy level given by

$$
\epsilon_{\mathrm{th}}=\epsilon_{c}-k T \ln \left(\frac{N_{c} C}{\omega}\right)
$$

plays an important role. ${ }^{8-11}$ Two energy regions separated by the thermalization energy $\epsilon_{\text {th }}$ are defined in conjunction with given frequency and temperature. In the shallower energy region where $\epsilon_{i}>\epsilon_{\text {th }}$, the normalized occupancy obeys a Boltzmann distribution

$$
\Delta \hat{f}_{i}=\exp \left(\frac{\epsilon_{c}-\epsilon_{i}}{k T}\right),
$$

indicating that a quasithermal equilibrium is established partially between electrons in the extended states and the associated localized states. On the other hand, for the deep localized states with energies $\epsilon_{i}<\epsilon_{\text {th }}$, the nonequilibrium occupation represented by

$$
\Delta \hat{f}_{i}=\frac{N_{c} C}{i \omega}
$$

occurs. A deeper thermalization energy appears for a lower frequency at a fixed temperature. The frequency-domain thermalization event corresponds to the progressively deeper trapping of carriers that causes dispersive transport observed in the time domain. The thermalization process in the presence of tunneling transitions between localized states will be examined in the following part of this section by means of numerical calculation.

The calculations are based on the density of states (DOS), which has been introduced to describe the conduction band tail, ${ }^{12,13}$

$$
\begin{aligned}
& D(\epsilon) \\
& =\left\{\begin{array}{l}
D_{c}\left(1-\frac{\epsilon_{c}-\epsilon}{\epsilon_{0}}\right) ; \epsilon>\epsilon_{c}-\epsilon_{0}+k T_{c}, \\
\frac{D_{c} k T_{c}}{\epsilon_{0}} \exp \left[-\left(1+\frac{\epsilon_{c}-\epsilon-\epsilon_{0}}{k T_{c}}\right)\right] ; \epsilon<\epsilon_{c}-\epsilon_{0}+k T_{c}
\end{array} .\right.
\end{aligned}
$$

This distribution is composed of a linearly decreasing part from the conduction band edge $\epsilon_{c}$, followed by an exponentially decreasing part into the gap. A linear distribution extended above $\epsilon_{c}$ gives the effective density of states $N_{c}$ $=D_{c} k T\left(1+k T / \epsilon_{0}\right)$. In the calculation, the continuous DOS was replaced by a set of discrete levels with a spacing $\delta$, normally set at $10 \mathrm{meV}$, for which the simultaneous linear Eqs. (27) and (28) are solved numerically. It has been confirmed that the calculated result is not altered by using $\delta$ less than $10 \mathrm{meV}$. Parameters used in the calculations are $D_{c}$ $=2 \times 10^{21} \mathrm{~cm}^{-3} \mathrm{eV}^{-1}, \epsilon_{0}=100 \mathrm{meV}, C=10^{-8} \mathrm{~cm}^{3} \mathrm{~s}^{-1}$, and $\alpha=10 \AA$. The value of localization radius is compatible with the previous estimates. ${ }^{6,14}$ The same frequency prefactor $\nu$

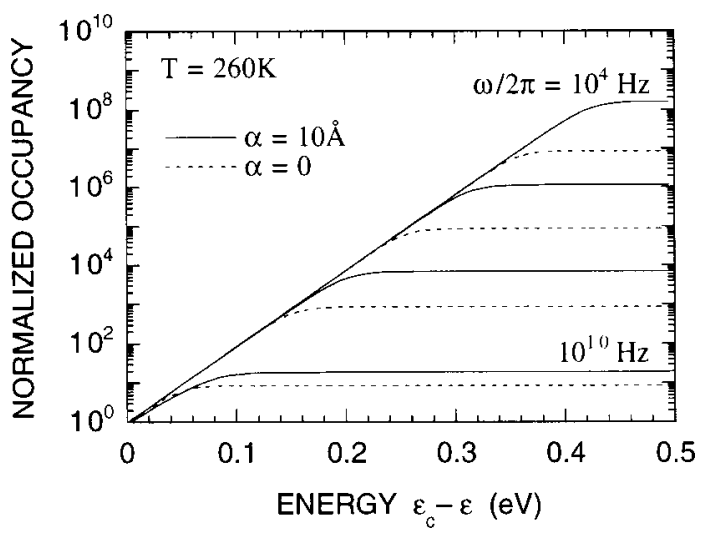

FIG. 1. Normalized occupation functions $\left|\Delta \hat{f}_{i}\right|$ for various frequencies $\omega / 2 \pi=10^{4}, 10^{6}, 10^{8}$, and $10^{10} \mathrm{~Hz}$ at a temperature $T=260 \mathrm{~K}$. Dashed and solid lines represent the results computed for $\alpha=0$ and $10 \AA$, respectively. The DOS distribution given by Eq. (36) with the characteristic temperature $T_{c}=260 \mathrm{~K}$ is assumed in the calculation.

$=N_{c} C$ for tunneling transitions and thermal emissions was assumed for simplicity, which would be reasonable so far as nonradiative tunneling transitions ${ }^{6}$ are concerned.

Figure 1 displays the absolute values of normalized occupancy $\left|\Delta \hat{f}_{i}\right|$ calculated as a function of energy for various frequencies. The occupation function for $\alpha=0$, plotted by dotted line as a reference, exhibits a sharp step at the thermalization energy, as interpreted previously on the basis of the analytical consideration. In the shallow energy region, the function behaves as an exponential, whereas uniform occupancy appears in the deep energy region. The thermalization energy shifts downward in the gap with decreasing frequency. Simultaneously, the deep nonequilibrium part becomes more prominent. Similar qualitative features can be found for the results obtained by assuming $\alpha=10 \AA$, shown by solid lines. It can be seen in the figure that tunneling interactions between localized states bring about a deep thermalization energy as well as an increased deep nonequilibrium occupancy. In this case, the energy at which the total escape rate described by $e_{i}+\sum_{j \neq i} C_{i j} N_{j}$ coincides with the frequency. $\omega$ is a good approximation to the thermalization energy that divides the exponential occupancy from the uniform occupancy. Figure 2 shows the normalized occupation functions calculated for various temperatures. As temperature decreases, the thermalization energy moves upward. The intensity of deep nonequilibrium occupancy varies slightly with temperature for $\alpha=0$, due to the attempt-to-escape frequency $N_{c} C$ being weakly dependent on temperature. In the presence of tunneling interactions, on the other hand, a strong temperature dependence emerges. The probability of tunneling transitions depends on the density of accessible states, as explicitly indicated by Eq. (21), so that the thermalization process is markedly influenced by the DOS distribution. This is in sharp contrast to the strong localization limit present without such a correlation. It is logical to expect that the thermalization of trapped electrons is accelerated for a higher DOS. This is confirmed by the calculations with varying the slope of exponential band tail.

Figure 3 illustrates the frequency resolved spectra of drift mobility amplitude $|\bar{\mu}|$ computed for various tempera- 


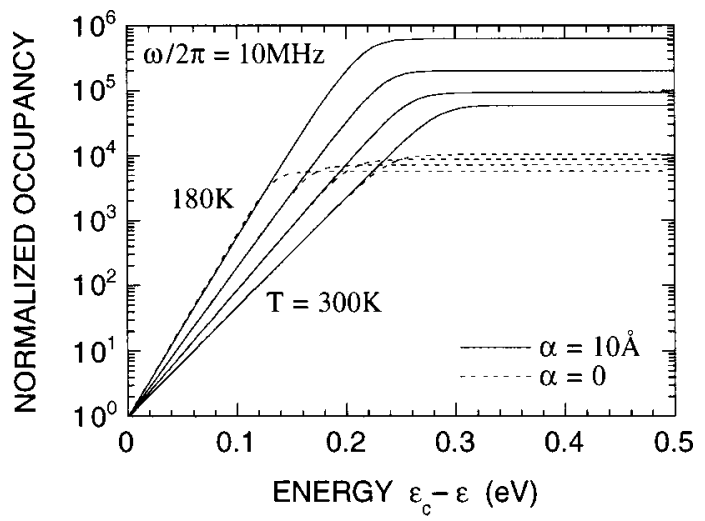

FIG. 2. Normalized occupation functions $\left|\Delta \hat{f}_{i}\right|$ for various temperatures $T$ $=180,220,260$, and $300 \mathrm{~K}$ at a frequency $\omega / 2 \pi=10^{6} \mathrm{~Hz}$. Dashed and solid lines represent the results computed for $\alpha=0$ and $10 \AA$, respectively. In the calculation, $T_{c}=260 \mathrm{~K}$ is assumed.

tures. The drift mobility behavior illustrated in the figure can be accounted for on the basis of the arguments on the normalized occupancy. At a certain temperature, the thermalization energy deepens with decreasing frequency, followed by the increasing population ratio of trapped electrons to free electrons $\Sigma_{i} \Delta n_{i} / \Delta n$ and thereby the decreasing magnitude of drift mobility. As temperature decreases, the drift mobility observed at any fixed frequency is reduced. In the absence of tunneling transitions, this phenomenon reflects mainly an expansion of the deep energy range available for electron trapping. The tunneling-assisted thermalization considerably enhances the deep nonequilibrium occupancy, yielding a significant lowering of drift mobility. The theoretical consideration given above, which only concerns absolute values of the associated quantities, is highly restricted but sufficient to gain a fundamental understanding of the MPC-derived drift mobility.

\section{EXPERIMENTAL DETAILS}

A $6-\mu$ m-thick sample of intrinsic $a$-Si:H deposited by glow discharge on a glass substrate at a substrate temperature of $200^{\circ} \mathrm{C}$ was used in the present study. The contacts were

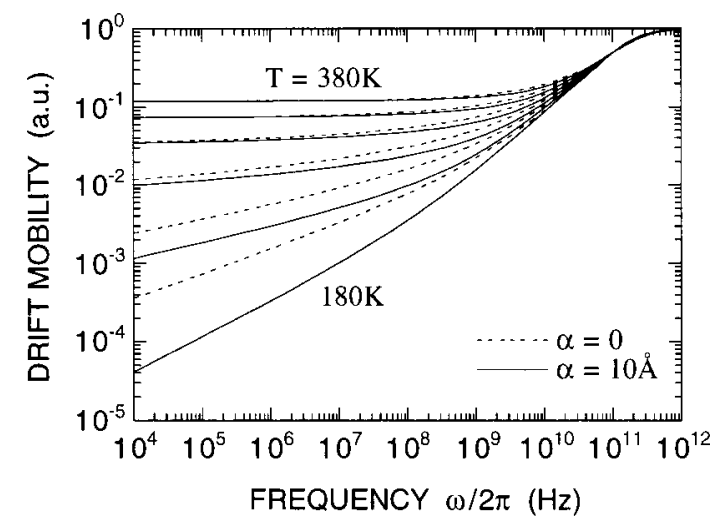

FIG. 3. Frequency dependence of normalized drift mobility $|\bar{\mu}| / \mu_{c}$ for various temperatures $T=180,220,260,300,340$, and $380 \mathrm{~K}$. Dashed and solid lines represent the results computed for $\alpha=0$ and $10 \AA$, respectively. In the calculation, $T_{c}=260 \mathrm{~K}$ is assumed. coplanar $\mathrm{Al}$ electrodes with a gap of $1 \mathrm{~mm}$ deposited on top of the sample. In the MPC experiments, the sample was mounted in a temperature-controlled cryostat. The measurement temperature was varied between 160 and $380 \mathrm{~K}$. The sample was uniformly illuminated by a $679 \mathrm{~nm}$ light from a diode laser. The light intensity was modulated sinusoidally at a frequency $\omega=2 \pi f$ with $f$ varying between $10 \mathrm{~Hz}$ and 40 $\mathrm{MHz}$. The highest frequency corresponds to a time scale of 4 $\mathrm{ns}$, which is comparable to the shortest time resolvable in transient photocurrent experiments. The electric field applied between the electrodes was typically $E=10 \mathrm{kV} / \mathrm{cm}$. At a frequency higher than $f=10 \mathrm{kHz}$, the amplitude of MPC $|\Delta \sigma|$ was detected by a rf spectrum analyzer and a wideband preamplifier. A standard lockin amplifier was also employed for low frequency measurements. The magnitude of drift mobility was determined by using the relation

$$
|\bar{\mu}|=\frac{\omega|\Delta \sigma|}{q \Delta G} .
$$

Prior to the drift mobility measurement, a linear response assumed in the theoretical analysis of MPC has been tested experimentally by varying the modulation amplitude $\Delta G$ while keeping the steady-state background intensity $G_{0}$ constant. The experiment actually showed excellent linearity over the modulation depths $\Delta G / G_{0}$ ranging from $1 \%$ to $100 \%$. All the data to be presented in this article were acquired in the linear response regime. Effects of the electric field applied between electrodes and the steady-state light intensity have also been carefully examined. It was found experimentally that an increase in high frequency MPC occurs to an extent that depends on power dissipation $\sigma_{0} E^{2}$ because of a thermally activated drift mobility. Consequently, the MPC-derived drift mobility decays appreciably following the light induced degradation of the steady-state photoconductivity. The apparent instability under light soaking can be eliminated by monitoring MPC with a sufficiently weak field and illumination. It has been confirmed from such measurements that light induced degradation of drift mobility is less than $10 \%$ after prolonged light exposure $(679 \mathrm{~nm}$, $18 \mathrm{~mW} / \mathrm{cm}^{2}, 7 \mathrm{~h}$ ). Further details of the light-soaking experiments will be given in a separate article. In the following section, the results obtained for the light-soaked sample with a negligible heating effect throughout a series of measurements are presented and discussed.

\section{RESULTS AND DISCUSSION}

Figure 4 displays the frequency dependence of MPC amplitude measured at various temperatures. These plots show a plateau in the lower frequency region, while in the higher frequency region they go downward with frequency. The low frequency behavior is indicative of the constant mobilitylifetime product that is expected for a quasiequilibrated transport system. On the other hand, the high frequency MPC is considered to be drift mobility controlled. Figure 5 illustrates the drift mobility spectra derived from the MPC data, which consist of two branches separated by a knee. Note that the translation into drift mobility can be validated 


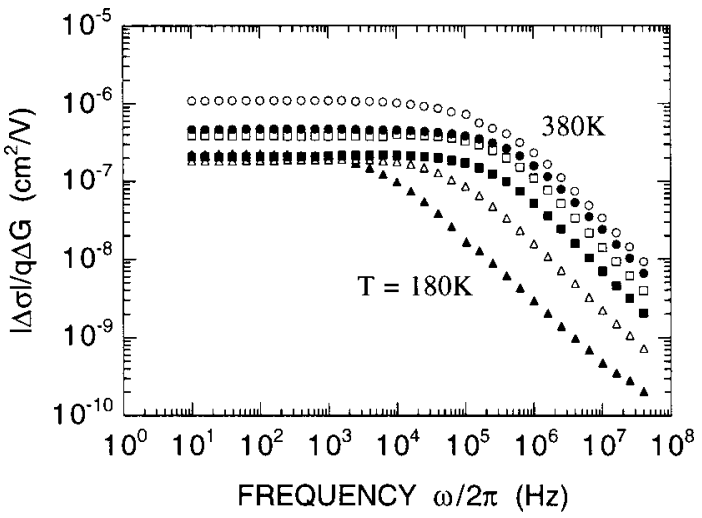

FIG. 4. Frequency dependence of normalized MPC amplitude $|\Delta \sigma| / q \Delta G$ measured for various temperatures $T=180,220,260,296,340$, and $380 \mathrm{~K}$.

only for the high frequency branch. The low frequency branch, showing a linear increase with frequency, merely represents $\bar{\mu} \bar{\tau}$ multiplied by frequency.

A direct way to investigate transport processes involved is to quantitatively compare the experimental data with the theoretical results. To begin with, the traditional multiple trapping model that ignores carrier interactions between localized states is examined. The temperature dependence of drift mobility measured at $f=10 \mathrm{MHz}$ is plotted in Fig. 6, together with the theoretical plots based on the DOS distributions given by Eq. (36) with various characteristic temperatures $T_{c}$. The experimental data exhibit a relatively steep decrease for lower temperatures, which significantly depart from the model calculations. Other probable types of DOS including a single exponential distribution, ${ }^{1}$ have also been considered for this assessment. These alternatives fail to reproduce the drift mobility behavior experimentally observed. In contrast, the transport model that takes tunneling interactions into consideration successfully accounts for the drift mobility data. The result of calculation using the DOS distribution given by Eq. (36) is plotted with the experimental data in Fig. 7. In the calculation, the extended-state mobility, the characteristic temperature, and the localization radius were chosen as $\mu_{c}=15 \mathrm{~cm}^{2} / \mathrm{V} \mathrm{s}, T_{c}=220 \mathrm{~K}$, and $\alpha=10$

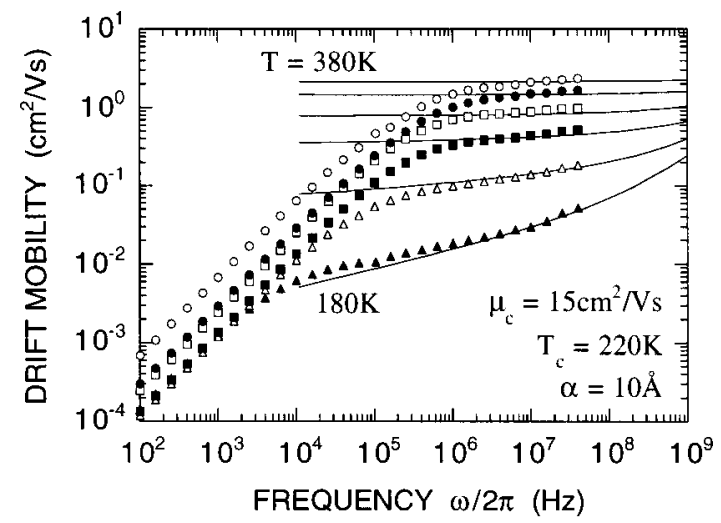

FIG. 5. Frequency resolved spectra of drift mobility calculated by using the relation $|\bar{\mu}|=\omega|\Delta \sigma| / q \Delta G$ from the MPC data shown in Fig. 4. Solid lines are theoretical fits with $\mu_{c}=15 \mathrm{~cm}^{2} / \mathrm{V} \mathrm{s}, T_{c}=220 \mathrm{~K}$, and $\alpha=10 \AA$. The DOS distribution given by Eq. (36) is assumed in the calculation.

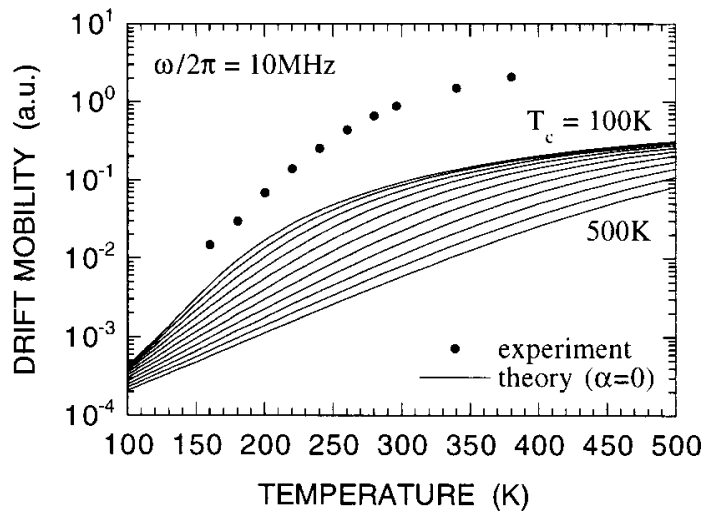

FIG. 6. Comparison of experimentally observed temperature dependence of $10 \mathrm{MHz}$ drift mobility (closed circles) to theoretical predictions from the multiple trapping model, assuming various conduction band tail slopes (solid lines).

$\AA$, respectively. Good agreement is also confirmed for the drift mobility spectra shown in Fig. 5 where the theoretical plots are drawn by solid lines.

The extended-state mobility evaluated here does not contradict the previous estimate, $\mu_{c}=10-15 \mathrm{~cm}^{2} / \mathrm{V} \mathrm{s},{ }^{6}$ whereas the characteristic temperature $T_{c}=220 \mathrm{~K}$ is low as compared with those implied from the TOF experiments, $T_{c}=250-300 \mathrm{~K} \cdot{ }^{1,13}$ It should be noticed, however, that the previous evaluation relies on the multiple trapping model constructed with the simplification that $\alpha=0$. This predicts a pretransit TOF current that shows a power-law time dependence $t^{\beta}$, with a temperature dependent slope $\beta=-(1$ $-T / T_{c}$ ) for $T<T_{c} .{ }^{1}$ The temperature dependence of the slope $\beta$ observed in low-field TOF experiments ${ }^{13,15}$ is summarized in Fig. 8. It is found in the figure that the theoretical prediction is valid only in a medium temperature range around $200 \mathrm{~K}$, leading to $T_{c}=260 \mathrm{~K}$. The sizable effect of tunneling interactions demonstrated by the present measurements tempts us to reexamine the time-domain results. In Appendix C, TOF solutions in the pretransit time regime are obtained quantitatively, in association with the transient drift mobility concept. The simulated result with characteristic temperature $T_{c}=230 \mathrm{~K}$ gives the best fit to the experimental

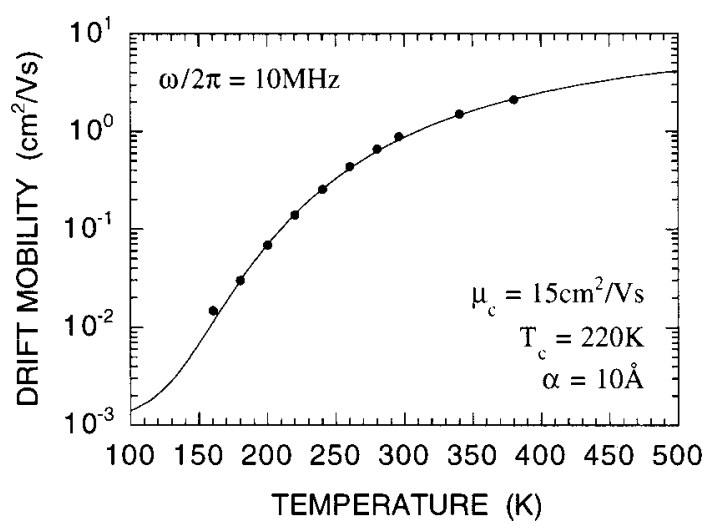

FIG. 7. Theoretical fit (solid lines) to temperature dependence of drift mobility $|\bar{\mu}|$ measured at frequency $\omega / 2 \pi=10 \mathrm{MHz}$ (closed circles). In the calculation, $\mu_{c}=15 \mathrm{~cm}^{2} / \mathrm{V} \mathrm{s}, T_{c}=220 \mathrm{~K}$, and $\alpha=10 \AA$ are assumed. 


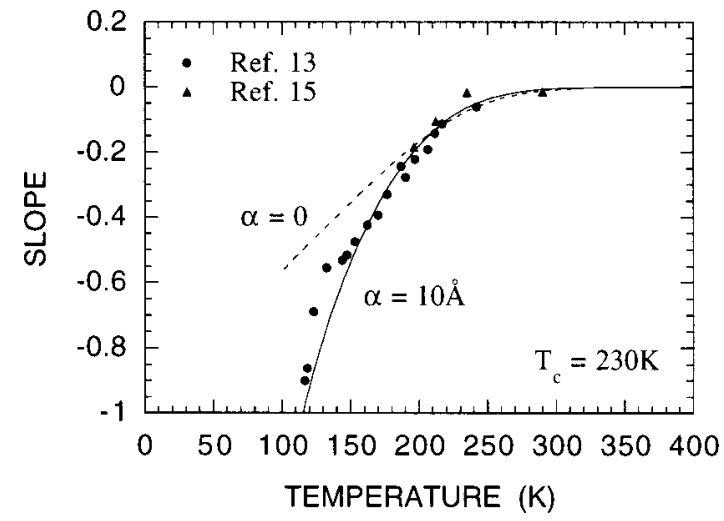

FIG. 8. Temperature-dependent slope $\beta$ of transient drift mobility decay. Low-field TOF data for $a$-Si:H $p-i-n$ diodes (Refs. 13 and 15) are shown by symbols. Dashed and solid lines represent the results computed for $\alpha=0$ and $10 \AA$, respectively. The DOS distribution given by Eq. (36) with $T_{c}$ $=230 \mathrm{~K}$ is assumed in the calculation.

data over a wide temperature range, as shown in Fig. 8. The estimation is slightly larger but comparable to the MPC result.

The MPC experiment has long been an attractive means of probing DOS distribution. Before concluding this section, we must address its applicability. Assuming the MPC transport is controlled by trapping and release from isolated localized states, the spectroscopic method is devised with a low temperature approximation ${ }^{9-11}$ such that

$$
D(\epsilon)=\frac{2}{\pi k T C}\left\{\operatorname{Im}\left[\frac{q \mu_{c} \Delta G}{\Delta \sigma}\right]-\omega\right\},
$$

where the energy scale is given by the thermalization energy

$$
\epsilon=\epsilon_{c}-k T \ln \left(\frac{N_{c} C}{\omega}\right) .
$$

Again, the problem lies in a finite localization radius that allows tunneling interactions to take a part in the MPC process, throwing doubt on the DOS reconstructed by means of the above formulas. To examine this, a numerical simulation has been carried out for the band tail distribution with $T_{c}$ $=220 \mathrm{~K}$. The results of the reconstruction are displayed in Fig. 9. A slight deviation from the assumed DOS for $\alpha=0$ is simply a result of thermal blurring effect. The figure clearly shows that the disagreement is greatly emphasized for $\alpha=10$ $\AA$, indicating an insufficient accuracy in the DOS deduced from MPC.

\section{SUMMARY}

Frequency-domain measurements of drift mobility of photocarriers by means of MPC technique have been applied to explore transport process in $a$ - $\mathrm{Si}: \mathrm{H}$. Theoretically, MPC transport is represented by introducing average transport parameters for all the charge carriers without loss of generality. A high frequency MPC is discussed with special emphasis since it is purely drift mobility controlled, leading to a simple way to survey the transport mechanism. The frequency resolved drift mobility is quantitatively analyzed in accordance with a general model that considers possible tunneling inter-

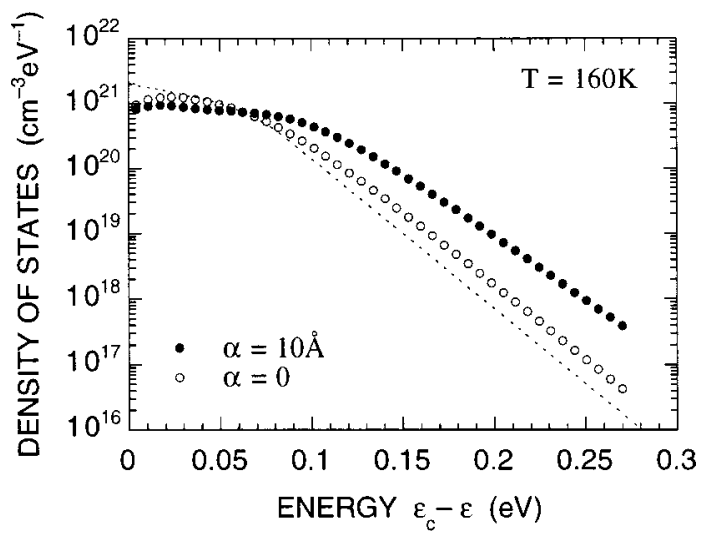

FIG. 9. DOS reconstructed by using Eq. (37) at a temperature $T=160 \mathrm{~K}$. Open and closed circles represent the results computed for $\alpha=0$ and $10 \AA$, respectively. The DOS distribution assumed in the calculation is plotted by dashed line.

actions between localized states in a mean field approach. Prime effects of incorporating tunneling transitions are viewed from the theoretical analysis as an accelerated thermalization of nonequilibrium carriers as well as a reduced magnitude of drift mobility. Using a finite localization length $\alpha=10 \AA$, the theory accounts well for MPC experiments performed for $a-\mathrm{Si}: \mathrm{H}$ over a wide range of frequency and temperature. The suggested incomplete localization is consistent with transient drift mobility data obtained from TOF experiments. The model consideration also reveals that the MPC methodology devised on the basis of isolated localized states yields an incorrectly mapped DOS distribution.

\section{APPENDIX A: RECOMBINATION}

Our theory for drift mobility measurements assumes a particular frequency that characterizes MPC behavior. The assumed changeover in frequency domain is here elucidated by inclusion of the monomolecular recombination mechanism. This is formally achieved by appending onto Eqs. (27) and (28) additional terms $\Delta n / \tau$ and $\Delta n_{i} / \tau_{i}$, respectively. For simplicity, we consider a tunneling recombination consisting of downward tunneling transitions of band tail electrons to holes deeply trapped in the gap, to which an energyindependent $\tau_{i}$ would be adapted reasonably. The results of calculation incorporating the recombination processes described above are illustrated in Fig. 10. The simulated spectra show two branches separated by a knee. It is readily found that in the high frequency branch, the spectrum is not affected by the recombination. We have confirmed that varying lifetime parameters shifts the frequency at which the knee appears while the high frequency branch stays unchanged. These observations lend credence to the present interpretation of MPC-derived drift mobility.

\section{APPENDIX B: HOPPING MOBILITY}

The mean transition rate of electrons from an occupied level $i$ to an unoccupied level $j$ with density $N_{j}\left(\ll n_{j}\right)$ is written by

$$
\left\langle w_{i j}\right\rangle=C_{i j} N_{j} .
$$




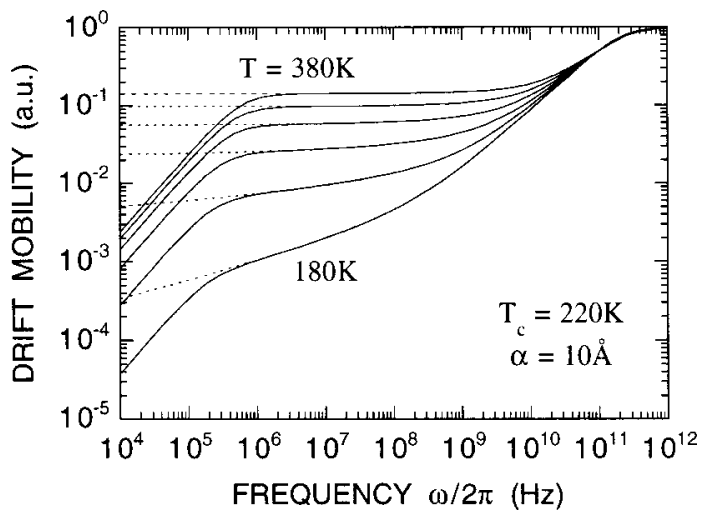

FIG. 10. Drift mobility spectra simulated by using the relation $\mid \bar{\mu}$ $=\omega|\Delta \sigma| / q \Delta G$ for various temperatures $T=180-380 \mathrm{~K}$. Solid lines represent the results computed by taking into account monomolecular recombination. The lifetime parameters are selected at $\tau=\left(C N_{r}\right)^{-1}$ and $\tau_{i}$ $=\left(\nu \pi \alpha^{3} N_{r}\right)^{-1}$ with $N_{r}=10^{15} \mathrm{~cm}^{-3}$. Dashed lines are plots calculated for $1 / \tau=1 / \tau_{i}=0$. The DOS distribution given by Eq. (36) with the characteristic temperature $T_{c}=220 \mathrm{~K}$ is assumed in the calculation.

Generally, there exists a particular level $j=m$ for which the rate $\left\langle w_{i j}\right\rangle$ is a maximum. The corresponding energy $\epsilon_{m}$ turns out to be

$$
\epsilon_{m}=\left\{\begin{array}{cc}
\epsilon_{c}-\epsilon_{0}+k T ; & \epsilon_{i}<\epsilon_{c}-\epsilon_{0}+k T \text { and } T>T_{c}, \\
\epsilon_{i} ; & \text { otherwise }
\end{array}\right.
$$

for the DOS line shape introduced in Sec. II C. The most probable transition considered here may give the largest contribution to electron transport via localized states. The diffusion coefficient for an isotropic hopping motion is then evaluated in the mean field approach to be

$$
D_{c i}=\frac{1}{6}\left\langle w_{i m}\left|\mathbf{r}_{m}-\mathbf{r}_{i}\right|^{2}\right\rangle \cong \frac{\alpha^{2}}{2} C_{i m} N_{m} .
$$

Applying the Einstein relationship, we have the mobility of electrons situated at a level $i$ expressed by

$$
\mu_{c i}=\frac{q \alpha^{2}}{2 k T} C_{i m} N_{m} .
$$

The contribution to the drift mobility in MPC transport is therefore represented by

$$
\bar{\mu}_{\text {hop }}=\frac{\sum_{i} \mu_{c i} \Delta n_{i}}{\Delta N} .
$$

The hopping mobility given above can be assessed from the numerical solution of the rate Eqs. (27) and (28). The results of calculation for $\alpha=10 \AA$ and $T_{c}=220 \mathrm{~K}$ are shown in Fig. 11. The computed hopping mobility, which is smaller by orders of magnitude than the measured mobility plotted in Fig. 5, supports the validity of the present analysis which neglects hopping transport.

\section{APPENDIX C: TRANSIENT DRIFT MOBILITY}

Here, we shall consider electron transport in a TOF configuration, for instance, a $p-i-n$ diode. The continuity equation is expressed by

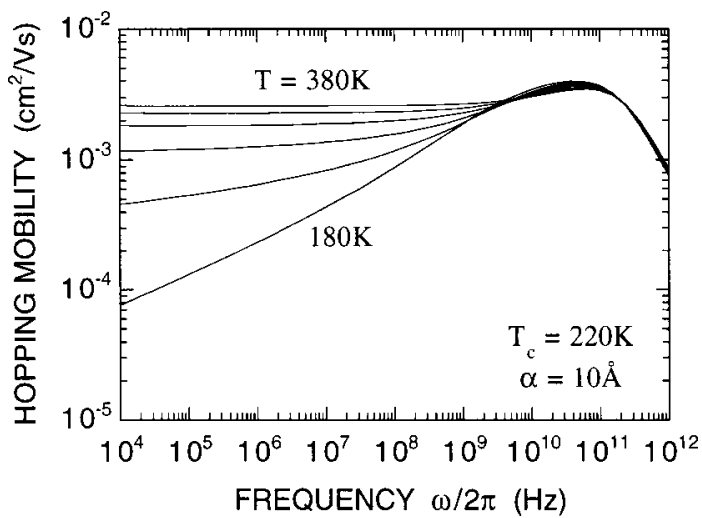

FIG. 11. Frequency dependent hopping mobility $\left|\bar{\mu}_{\text {hop }}\right|$ calculated for various temperatures $T=180-380 \mathrm{~K}$. In the calculation, $T_{c}=220 \mathrm{~K}$ is assumed.

$$
\frac{\partial}{\partial t} N+\frac{\partial}{\partial x} \mu_{c} n E=0,
$$

for the one-dimensional transport from $x=0$ to $x=d$. Evidently, the charge injected at $t=0$ is retained within the sample volume before the extraction at $x=d$ starts. An explicit representation for the charge conservation;

$$
\frac{\partial}{\partial t} \tilde{N}(t)=0
$$

can be directly derived from Eq. (C1), where $\tilde{N}$ denotes the spatial average defined by the integration from $x=0$ to $d$ divided by $d$. The pretransit TOF current $J(t)$ observed under a uniform electric field is therefore related to the transient drift mobility as

$$
\bar{\mu}(t)=\mu_{c} \frac{\tilde{n}(t)}{\tilde{N}(0)}=\frac{J(t) d}{Q E},
$$

where $Q=q \tilde{N}(0) d$ refers to the injected charge. The expression coincides with that deduced previously. ${ }^{16}$ In the smallsignal TOF experiment, the trap saturation can be avoided so that the concentrations of electrons in the extended states $\tilde{n}$ and in the localized states $\tilde{n}_{i}$ obey the linearized rate equations

$$
\begin{aligned}
& \frac{d \tilde{n}}{d t}=-\sum_{i}\left(\tilde{n} C N_{i}-e_{i} \tilde{n}_{i}\right), \\
& \frac{d \tilde{n}_{i}}{d t}=\tilde{n} C N_{i}-e_{i} \tilde{n}_{i}-\tilde{n}_{i} \sum_{j \neq i} C_{i j} N_{j}+N_{i} \sum_{j \neq i} \tilde{n}_{j} C_{j i} .
\end{aligned}
$$

We have to keep in mind that the discussion ignores possible contributions of hopping transport. A timedependent hopping mobility can be defined as

$$
\bar{\mu}_{\text {hop }}(t)=\frac{\sum_{i} \mu_{c i} \tilde{n}_{i}(t)}{\tilde{N}(0)} .
$$

Our preliminary calculation has revealed the trivial role played by hopping mobility in determining the transient photocurrents, at least for temperatures above $100 \mathrm{~K}$. However, it is known experimentally that even at a temperature around $200 \mathrm{~K}$, the transient photocurrent measured for $a-\mathrm{Si}: \mathrm{H}$ is significantly modified when an electric field exceeding 


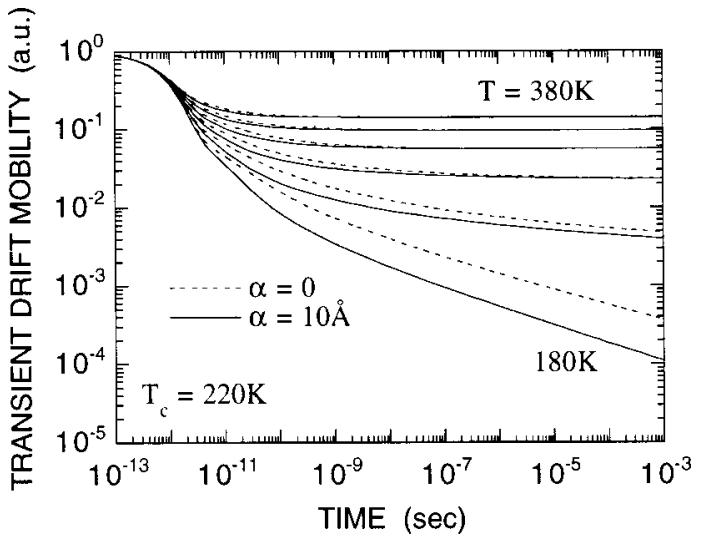

FIG. 12. Time dependent drift mobility $\bar{\mu}(t)$ for various temperatures $T$ $=180-380 \mathrm{~K}$. Dashed and solid lines represent the results computed for $\alpha=0$ and $10 \AA$, respectively. In the calculation, $T_{c}=220 \mathrm{~K}$ is assumed.

$10^{5} \mathrm{~V} / \mathrm{cm}$ is applied, due to a field-enhanced band-tail hopping. ${ }^{13}$ The present model does not include the fieldrelated effect and concerns the low-field transient drift mobility.

Figure 12 shows the transient drift mobility $\bar{\mu}(t)$ obtained from numerically solving the differential Eqs. (C4) and (C5) for the DOS given by Eq. (36). The decay of transient drift mobility follows approximately a power law time dependence like $t^{\beta}(\beta<0)$ for long times. Note that such a long time portion can be usually observed in the experiments. A faster decay in the presence of tunneling interactions is interpreted as being due to a faster thermalization, which is confirmed from the time evolution of occupation function simultaneously computed.

${ }^{1}$ T. Tiedje, in Semiconductors and Semimetals, edited by J. I. Pankove (Academic, New York, 1984), Vol. 21C, pp. 207-238.

${ }^{2}$ F. W. Schmidlin, Phys. Rev. B 16, 2362 (1977).

${ }^{3}$ D. Ritter, E. Zeldov, and K. Weiser, Phys. Rev. B 38, 8296 (1988).

${ }^{4}$ K. Hattori, H. Okamoto, and Y. Hamakawa, Phys. Rev. B 45, 1126 (1992).

${ }^{5}$ Y. Tang and R. Braunstein, J. Appl. Phys. 79, 850 (1996).

${ }^{6}$ R. A. Street, in Hydrogenated Amorphous Silicon, edited by R. W. Cahn, E. A. Davis, and I. M. Ward, Cambridge Solid State Science Series (Cambridge University Press, Cambridge, England, 1991).

${ }^{7}$ M. Abraham and V. Halpern, Philos. Mag. B 62, 537 (1990).

${ }^{8}$ H. Oheda, J. Appl. Phys. 52, 6693 (1981).

${ }^{9}$ R. Bruggemann, C. Main, J. Berkin, and S. Reynolds, Philos. Mag. B 62, 29 (1990).

${ }^{10}$ C. Longeaud and J. P. Kleider, Phys. Rev. B 45, 11672 (1992).

${ }^{11}$ K. Hattori, Y. Adachi, M. Anzai, H. Okamoto, and Y. Hamakawa, J. Appl. Phys. 76, 2841 (1994).

${ }^{12}$ R. A. Street, J. Kakalios, and M. Hack, Phys. Rev. B 38, 5603 (1988).

${ }^{13}$ C. E. Nebel, R. A. Street, N. M. Johnson, and J. Kocka, Phys. Rev. B 46, 6789 (1992).

${ }^{14}$ K. Hattori, Y. Ota, K. Sato and H. Okamoto, J. Appl. Phys. 84, 4974 (1998).

${ }^{15}$ J. Kocka, O. Klima, E. Sipek, C. E. Nebel, G. H. Bauer, G. Juska, and H. Hoheisel, Phys. Rev. B 45, 6593 (1992).

${ }^{16}$ E. A. Schiff, in Tetrahedrally Bonded Amorphous Semiconductors, edited by D. Adler and H. Fritzsche, Institute for Amorphous Semiconductor Series (Plenum, New York, 1985), pp. 357-366. 